\title{
How to set up a Hydroxychloroquine Retinopathy Screening Service
}

\author{
Farhan H. Zaidi ${ }^{1,2,3}$ - Christina A. Rennie ${ }^{1,2} \cdot$ Amie K. Drinkwater $^{2} \cdot$ Debendra Sahu $^{1,2} \cdot$ Engin Akyol $^{2}$. \\ Andrew J. Lotery $\mathbb{( i D}^{1,2,4}$
}

Received: 11 February 2019 / Accepted: 22 February 2019 / Published online: 26 April 2019

(c) The Royal College of Ophthalmologists 2019

\begin{abstract}
A major development in clinical practice has been new guidance from the Royal College of Ophthalmologists (RCOphth) on the risk of retinal toxicity from Hydroxychloroquine (HCQ) and Chloroquine [1]. The risk was previously considerably underestimated - the incidence is now felt to be up to $7.5 \%$ after 5 years' use and $20-50 \%$ after 20 years' use [1]. Much of the evidence for these estimates come from the US [2]. Early UK data is cited herein. Ophthalmologists are highlighting the need for greater awareness of these risks, and cases of missed retinopathy are appearing in the courts [3-7].

Having received a number of requests for advice on screening from NHS ophthalmology departments we provide our expertise from University Hospital Southampton (UHS), a major NHS university teaching hospital which has implemented the new RCOphth recommendations. UHS has about 1500 adults and 25 children on hydroxychloroquine.
\end{abstract}

\section{Assess the size of the eligible population}

Responsibility for starting, stopping and referring patients on HCQ for screening rests with the prescribing physician. Chloroquine is rarely prescribed. HCQ is commonly

Farhan H. Zaidi

fhz12@hotmail.com

1 Medical Retina Service, Department of Ophthalmology, University Hospital Southampton NHS Foundation Trust, Southampton, UK

2 Department of Ophthalmology, University of Southampton NHS Foundation Trust, Southampton, UK

3 Eye Emergency Department, University of Southampton NHS Foundation Trust, Southampton, UK

4 Faculty of Medicine, University of Southampton, Southampton, UK prescribed, being in National Institute for Clinical Excellence (NICE) guidance for rheumatoid arthritis, lupus and photosensitive drug eruptions. Use is increasing [1]. Most prescriptions are initiated by rheumatologists, dermatologists, and sometimes paediatricians. Contact these physicians for an estimate of how many patients are on HCQ. Pharmacists may help, though many patients obtain repeat prescriptions from their General Practitioners (GPs).

\section{Commissioning the service}

Team up with your managerial and imaging colleagues to formulate a business plan based on existing numbers, new starters per year, how many stop HCQ, imaging capacity and staffing costs. While negotiating with Clinical Commissioning Groups (CCGs) provide a bundled tariff for seeing patients in virtual clinics and reviews with Optical Coherence Tomography (OCT), Fundus Autofluorescence (AF), Visual Fields and support staff. It may help reminding commissioners that patients on HCQ have often been brought into Medical Retina (MR) clinics for decades - the screening process formalises this into a protocol.

\section{Triage and E-Referral}

Any patient with visual symptoms is urgently brought into face-to-face clinics. All other referrals are made by the prescribing physician via the e-Referral system which we incorporated onto Charts (E-Quest) as a Specialty Service Request (Charts/E-Quest is a leading EMIS software application (Egton Medical Information Systems) for electronic requests which is widely used in the NHS). Requests are received electronically by the Ophthalmology Department (Fig. 1), triaged and prioritised for review if they are high risk. 


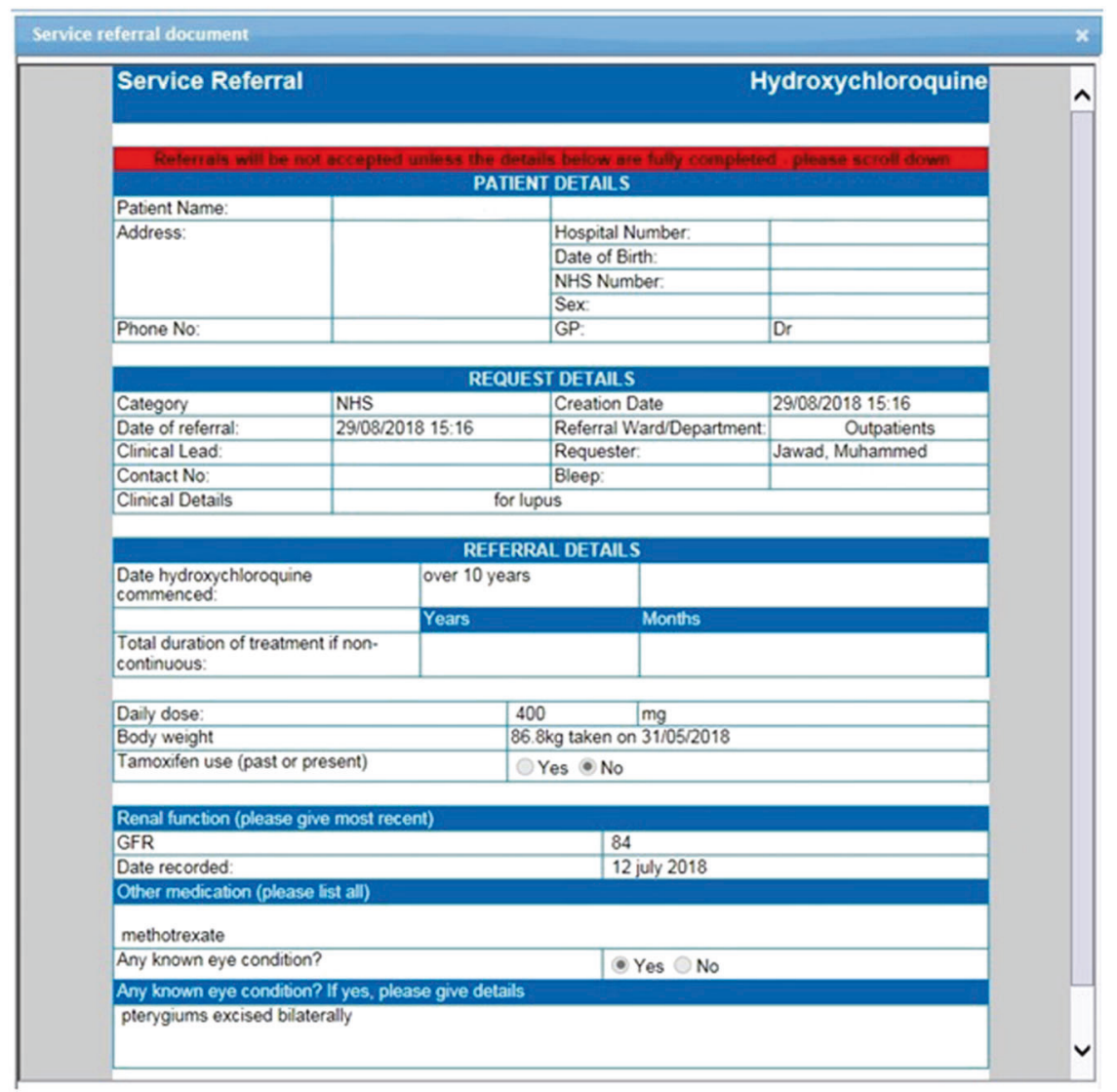

Fig. 1 An e-referral submitted by a physician and reviewed by the Ophthalmology Department on Charts / E-Quest (a leading EMIS software application (Egton Medical Information Systems)) before triaging. The e-Referral can be set up by working with your IT colleagues and facilitates the acquisition of data on High Risk Characteristics (HRC) from referring physicians who must input these parameters when referring e.g. weight, dose, estimated Glomerular Filtration Rate (eGFR) (confers high risk if $<60 \mathrm{ml} / \mathrm{min} / 1.73 \mathrm{~m}^{2}$ ), treatment duration (high risk if $>5$ years), tamoxifen use. Dose is obtained by the triaging clinician dividing dose by weight in $\mathrm{mg} / \mathrm{kg}$ (high risk doses are $>5 \mathrm{mg} / \mathrm{kg} / \mathrm{day}$ ) though could also be electronically calculated. Triaging using HRC allows stratification of risk into high risk (one or more of these risk factors) versus low risk referrals and is useful for both clinical and research purposes. Any patient with significant visual symptoms is brought urgently into clinic

\section{Virtual clinics}

Patients are sent an information leaflet. Virtual clinics accommodate 30 patients in $4 \mathrm{~h}$ sessions. We use one nurse, 3-4 healthcare assistants and 3-4 imaging technicians. Patients have a Visual Acuity (logMAR) recorded on Medisoft (an Electronic Patient Record (EPR), Humphrey automated 10-2 Visual Field, pupil dilation, Heidelberg OCT and blue field FAF with $55^{\circ}$ lens allowing imaging of extra-macular areas (not all OCT manufacturers offer this) [Heidelberg Spectralis OCT1 and OCT2]. HCQ toxicity may present first as extra-macular hyperfluorescence. The $55^{\circ}$ lens may not be feasible in all units but several standard autofluorescent images can achieve the same peripheral coverage.

\section{Virtual reviews}

At present reviews are conducted by experienced consultants with Medical Retina expertise (FHZ, CAR), as the consequences of missing toxicity are potentially significant and expertise limited. It is easy to miss subtle toxicity. New features are being described such as outer photoreceptor layer (OPL) thinning (Marmor, personal communication; Melles, personal communication) [8]. We noted using high resolution Heidelberg imaging strikingly regular focal OPL swelling with thinning of the outer nuclear layer (ONL) in the peri-central macula of high risk patients [FHZ, CAR, AJL]. Even considering the optical properties of Henle's layer and variability in the imaging angle, the regularity was intriguing. At present we suggest this can be a non-specific 


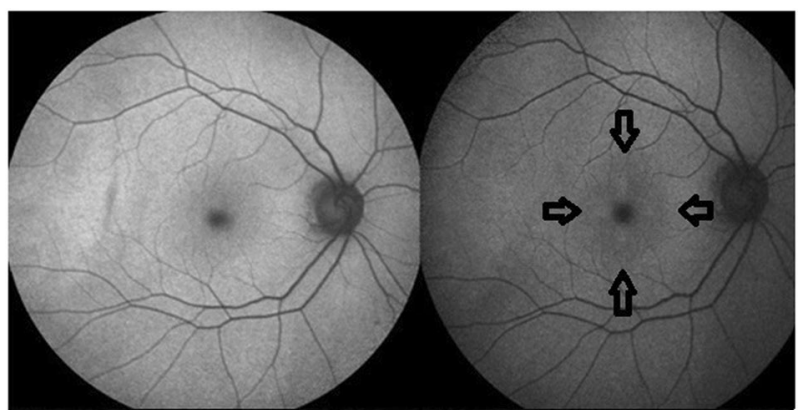

Fig. 2 Top left shows blue field FAF pattern using Heidelberg HRA-1 (which uses a spot target) and top right using HRA-2 (which uses a cross target) with corresponding 'Sign of the Cross' artefact on autofluorescence peri-foveally within the arrows. [Heidelberg Spectralis OCT1 and OCT2]

finding of mild HCQ toxicity. We also found artefacts in a FAF 'Sign of the Cross' sign originating from the cross target used in the Heidelberg HRA-2 OCT scanner (though not in HRA-1 which uses a spot target) (Fig. 2).

Patients graded with definite toxicity on OCT/FAF are brought to urgent face-to-face review. Unreliable fields are repeated virtually. We have started use of mutifocal ERG (mfERG) in equivocal cases. In units without mfERG the ophthalmologist needs to decide on its feasibility as it is uncommonly available. The patient, patient's GP and referring physician are informed in writing of outcomes, facilitated by the imaging-clinic review tab (Medisoft). The critical decision to continue treatment or not is that of the prescribing physician.

\section{Research, audits and outcomes}

It is vital outcomes are audited. Crucially failsafe procedures need development so patient referrals and reviews are tracked to avoid screening pitfalls and clinical harm.

Early data from the first 51 patients screened at UHS $(1+$ HRC) found $22(43 \%)$ had initial suspicion of toxicity (mean Logmar VA 0.18, range 0-1.32), 25 (49\%) were screen negative (mean Logmar VA 0.12, range $0-0.24$ ), 3 were equivocal (mean Logmar VA 0.14, range 0-0.3) and 1 was unknown (co-existent macular pathology). Average treatment duration was 10.8 years in suspected Screen Positives versus 8.75 years in Screen Negatives. Average HCQ dose was 3.73 $\mathrm{mg} / \mathrm{kg}$ in suspected Screen Positives versus $3.5 \mathrm{mg} / \mathrm{kg}$ in Screen Negatives. Average age of suspected Screen Positives was 63 years and 57 years in Screen Negatives. In cases with suspicion of toxicity (which often seems 'mild') repeat OCT scanning is useful. This has started to show that for mild cases parafoveal OPL thinning is not always repeatable between scans, suggesting the appearance can often be due to variability in subject fixation between tests and density of OCT scanning with regard to imaging Henle's layer. However in other cases the appearances do seem repeatable and are convincing of potential toxicity. While only early estimates these results may be consistent with some US data $[1,2,8]$. Several in this first cohort were the highest risk patients at UHS, many on HCQ for well over 20 years.

\section{Children}

Most children at UHS are on HCQ for lupus, some at risk of renal impairment. Average dose is higher than adults at 6.5 $\mathrm{mg} / \mathrm{kg}$ body weight. We plan to attempt fields on patients aged $7+$ in virtual clinics. Younger children will be seen in paediatric ophthalmology clinics with orthoptist-perfomed VAs, OCT and FAF.

\section{Conclusions}

The new RCOphth guidelines on HCQ screening can be implemented into a busy NHS ophthalmology service with the use of a virtual pathway and imaging technicians. Early data corroborates some RCOphth estimates of toxicity in high risk groups, though toxicity may be mild. More screening of patients and data analysis is planned at UHS which will provide more data. As the resolution of OCT has advanced so has detection of mild retinopathy, which may in part explain why the risk was previously underestimated. The significance of 'mild' toxicity is unknown. Ophthalmologists need to support decision-making by prescribing physicians. HCQ retinal screening requires the formal allocation of staffing and equipment. HCQ screening is felt by some Clinical Commissioning Groups to place a potential burden on existing capacity. We have shown Hospital-based screening is practically feasible. In many regions community providers are being considered to help in reducing the strain on Hospital Eye Services and providing care closer to home e.g. diabetic retinopathy screening programmes, who also offer failsafe expertise. This demands suitably experienced retinal subspecialists who can interpret the potentially subtle imaging changes and train potential graders.

\section{Compliance with ethical standards}

Conflict of interest The authors declare that they have no conflict of interest.

Publisher's note: Springer Nature remains neutral with regard to jurisdictional claims in published maps and institutional affiliations.

\section{References}

1. The Royal College of Ophthalmologists. Hydroxychloroquine and Chloroquine Retinopathy: Recommendations on Screening. 
February 2018. https://www.rcophth.ac.uk/wp-content/uploads/ 2018/07/Hydroxychloroquine-and-Chloroquine-Retinopathy-

Screening-Guideline-Recommendations.pdf.

2. Melles RB, Marmor MF. The risk of toxic retinopathy in patients on long-term hydroxychloroquine therapy. JAMA Ophthalmol. 2014;132:1453-60.

3. Yusuf IH, Lotery AJ, Ardern-Jones MR. Joint recommendations for retinal screening in long-term users of hydroxychloroquine and chloroquine in the United Kingdom. Br J Dermatol. 2018;179:995-6. https://doi.org/10.1111/bjd.16782.

4. Yusuf IH, Ledingham JM, MacPhie E, Lotery AJ, Galloway J. Monitoring for retinal toxicity in patients taking hydroxychloroquine and chloroquine. Rheumatology. 2018;58:3-4. https:// doi.org/10.1093/rheumatology/key024.
5. Lotery AJ, Yusuf IH. Requirement for retinal screening in patients taking hydroxychloroquine and chloroquine. $\mathrm{Br} \mathrm{J}$ Gen Pract. 2018;68:120.

6. Alwitry A. Medico-legal: case in point - what can we learn from litigation? Hydroxychloroquine toxicity. Eye News. 2017;24: $52-3$.

7. Latasiewicz M, Gourier H, Yusuf IH, Luqmani R, Sharma SM, Downes SM. Hydroxychloroquine retinopathy: an emerging problem. Eye (Lond). 2017;31:972-6. https://doi.org/10.1038/eye. 2016.297.

8. Lally DR, Heier JS, Baumal C, Witkin AJ, Maler S, Shah CP, et al. Expanded spectral domain-OCT findings in the early detection of hydroxychloroquine retinopathy and changes following drug cessation. Int J Retina Vitreous. 2016;2:18. 